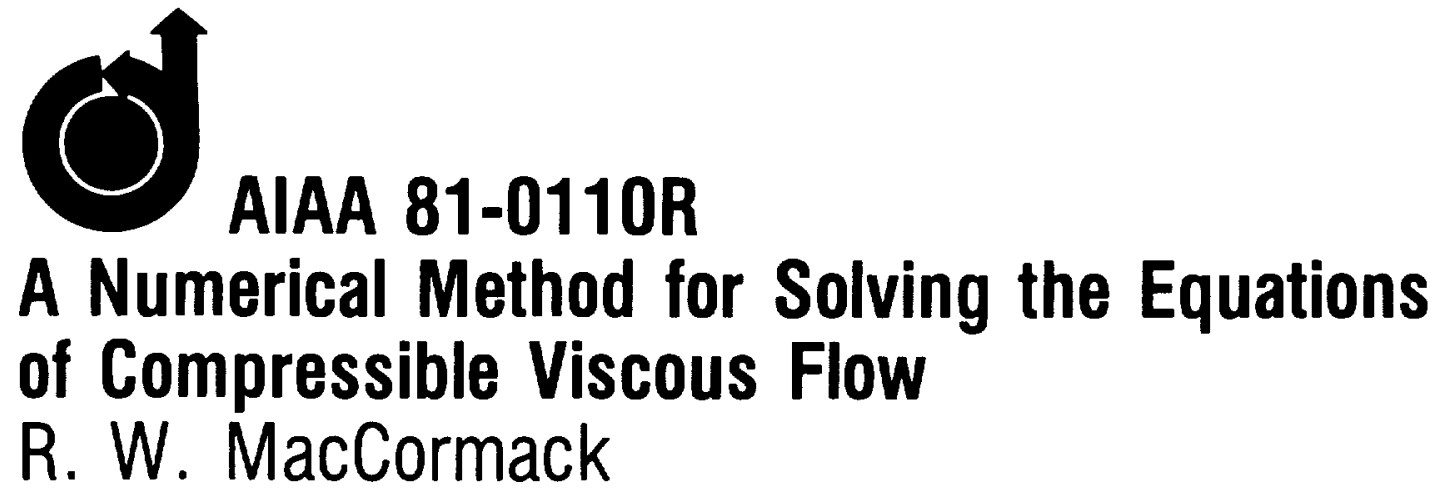

Reprinted from 


\title{
NASATM- $-82-208076$ \\ A Numerical Method for Solving the Equations of Compressible Viscous Flow
}

\author{
R. W. MacCormack* \\ NASA Ames Research Center, Moffett Field, Calif.
}

\begin{abstract}
Although much progress has already been made in solving problems in aerodynamic design, many new developments are still needed before the equations for unsteady compressible viscous flow can be solved routinely. This paper describes one such development. A new method for solving these equations has been devised that 1 ) is second-order accurate in space and time, 2) is unconditionally stable, 3) preserves conservation form, 4) requires no block or scalar tridiagonal inversions, 5) is simple and straightforward to program (estimated $10 \%$ modification for the update of many existing programs), 6) is more efficient than present methods, and 7) should easily adapt to current and future computer architectures. Computational results for laminar and turbulent flows at Reynolds numbers from $3 \times 10^{5} 103 \times 10^{7}$ and at CFL numbers as high as $10^{3}$ are compared with theory and experiment.
\end{abstract}

\section{Introduction}

T HE compressible form of the Navier-Stokes equations adequately describes aerodynamic flow at standard temperatures and pressures. If we could efficiently solve these equations, there would be no need for experimental tests to design flight vehicles or other aerodynamic devices. Although much progress has been made toward their numerical solution, the calculation of flowfields past complete aircraft configurations at flight Reynolds numbers is presently far beyond our reach. Such calculations await substantial progress in devising more powerful and reliable computer hardware, in further understanding and modeling the physics of turbulence, and in developing more accurate and efficient numerical methods. One development is described in this paper.

Several important developments have been made in numerical methods in recent years. Chief among them has been the development of noniterative implicit methods for solving the compressible Navier-Stokes equations. These methods, not subject to conventional explicit stability conditions, have significantly improved computational efficiency over the earlier explicit methods. However, their time step sizes are still frequently limited by severe accuracy and stability criteria, and their computer time per step, as well as their programming complexity, is much larger than that of the explicit methods. The goal of the present research is to develop a method for solving the compressible form of the Navier-Stokes equations at high Reynolds number that is unconditionally stable, computationally more efficient than existing methods, and simple and straightforward to program. The new method contains two stages. The first stage uses the explicit predictor-corrector finite-difference method presented by the author in 1969.' The generated finitedifference equations approximate the governing equations of fluid flow to second-order accuracy in space and time, are simple to program, but are subject to restrictive explicit stability conditions. The second stage removes these stability conditions by transforming numerically the equations of the first stage into an implicit form. The resulting matrix equations to be solved are either upper or lower block

Presented as Paper 81-0110 at the AIAA 19th Aerospace Sciences Meeting, St. Louis, Mo., Jan. 12-15, 1981; submitted March 18, 1981; revision received Jan. 5, 1982. This paper is declared a work of the U.S. Government and therefore is in the public domain.

-Senior Staff Scientist; presently Associate Professor, Dept. of Aeronautics and Astronautics, University of Washington, Seattle, Wash. Member AIAA. bidiagonal equations and are solved more easily than the block tridiagonal matrix equations of existing implicit methods. Although the method is presented in one and two dimensions, its extension to three dimensions is straightforward.

\section{Navier-Stokes Equations}

In two dimensions and by neglecting body forces and heat sources, the unsteady compressible form of the Navier-Stokes equations may be written in conservation form as

$$
\frac{\partial U}{\partial t}+\frac{\partial F}{\partial x}+\frac{\partial G}{\partial y}=0
$$

where

$$
\begin{aligned}
& U=\left[\begin{array}{l}
\rho \\
\rho u \\
\rho v \\
e
\end{array}\right] \\
& F=\left[\begin{array}{c}
\rho u \\
\rho u^{2}+\sigma_{x} \\
\rho u v+\tau_{x y} \\
\rho+\tau_{y x} v-k(\partial T / \partial x) \\
\rho v \\
\rho u v+\tau_{y x} \\
\rho v^{2}+\sigma_{y} \\
\left(e+\sigma_{x}\right)
\end{array}\right] \\
& G=\left[\begin{array}{c}
\tau_{x y} u-k(\partial T / \partial y)
\end{array}\right]
\end{aligned}
$$

and where

$$
\begin{aligned}
& \sigma_{x}=p-\lambda\left(\frac{\partial u}{\partial x}+\frac{\partial v}{\partial y}\right)-2 \mu \frac{\partial u}{\partial x} \\
& \tau_{x y}=\tau_{y x}=-\mu\left(\frac{\partial u}{\partial y}+\frac{\partial v}{\partial x}\right) \\
& \sigma_{y}=p-\lambda\left(\frac{\partial u}{\partial x}+\frac{\partial v}{\partial y}\right)-2 \mu \frac{\partial v}{\partial y}
\end{aligned}
$$


in terms of density $\rho, x$ - and $y$-velocity components $u$ and $v$, viscosity coefficients $\lambda$ and $\mu$, total energy per unit volume $e$, coefficient of heat conductivity $k$, and temperature $T$. Finally, the pressure $p$ is related to the specific internal energy $\epsilon$ and $\rho$ by an equation of state, $p(\epsilon, \rho)$, where $\epsilon=e / \rho-\left(u^{2}+v^{2}\right) / 2$.

\section{Basic Elements of the Numerical Method}

Before discussing solution of the complete Navier-Stokes equations, it is worthwhile to consider the basic elements of the method applied to the following model equation:

$$
\frac{\partial u}{\partial t}=-c \frac{\partial u}{\partial x}+\nu \frac{\partial^{2} u}{\partial x^{2}}
$$

The flow variable $u$ governed by this equation convects with speed $c$ and diffuses with kinematic viscosity $\nu$.

\section{Explicit Method}

The 1969 explicit predictor-corrector finite-difference equations, in delta form, for approximating Eq. (2) are

$$
\begin{aligned}
& p:\left\{\begin{array}{c}
\Delta u_{i}^{n}=-\frac{\Delta t c}{\Delta x}\left(u_{i+1}^{n}-u_{i}^{n}\right)+\frac{\Delta t v}{\Delta x^{2}}\left(u_{i+1}^{n}-2 u_{i}^{n}+u_{i-1}^{n}\right) \\
u_{i}^{\overline{n+1}}=u_{i}^{n}+\Delta u_{i}^{n}
\end{array}\right. \\
& c:\left\{\begin{array}{l}
\Delta u_{i}^{\overline{n+1}}=-\frac{\Delta t c}{\Delta x}\left(u_{i}^{\overline{n+1}}-u_{i-1}^{\overline{n+1}}\right)+\frac{\Delta t \nu}{\Delta x^{2}}\left(u_{i+1}^{n+1}-2 u_{i}^{n+1}+u_{i-1}^{n+1}\right) \\
u_{i}^{n+1}=1 / 2\left(u_{i}^{n}+u_{i}^{\overline{n+1}}+\Delta u_{i}^{\bar{n}+1}\right)
\end{array}\right.
\end{aligned}
$$

The first step predicts a new solution at time $t=(n+1) \Delta t$ at each mesh point $i$ from the known solution at time $t=n \Delta t$ and uses a one-sided difference to approximate the first derivative and a centered difference for the second derivative. The second step corrects the predicted values with an opposite onesided difference for the first derivative. The method is secondorder accurate and stable if the time step satisfies the following condition:

$$
\Delta l \leq \frac{1}{(c / \Delta x)+\left(2 v / \Delta x^{2}\right)}
$$

Implicit Method, $v=0$

If $c<0$ Eq. (2) may be approximated using the following first-order-accurate one-sided difference equation:

$$
u_{i}^{n+1}=u_{i}^{n}-(l-\alpha) \frac{c \Delta t}{\Delta x}\left(u_{i+1}^{n},-u_{i}^{n}\right)-\alpha \frac{c \Delta t}{\Delta x}\left(u_{i+1}^{n+1}-u_{i}^{n+1}\right)
$$

or

$$
\left(1+\frac{\lambda \Delta t}{\Delta x}\right) \delta u_{i}^{n+1}=-\frac{c \Delta t}{\Delta x} \Delta+u_{i}^{n}+\frac{\lambda \Delta t}{\Delta x} \delta u_{i+1}^{n+1}
$$

where

$$
\delta u_{i}^{n+1}=u_{i}^{n+1}-u_{i}^{n}, \quad \Delta_{+} u_{i}^{n}=u_{i+1}^{n}-u_{i}^{n}, \quad \lambda=\alpha|c|
$$

The parameter $\lambda$ was chosen so that the above two implicit equations would be identical. These equations are unconditionally stable (for $c \leq 0$ ) if the implicit blending parameter $\alpha$ is greater than $1 / 2$. From now on in this paper, however, we will choose $\lambda$ in a more general way to ensure only unconditional stability (according to linear stability theory). Accordingly, for stability we choose

$$
\lambda \geq \frac{l}{2}\left(|c|-\frac{\Delta x}{\Delta t}\right)
$$

The unsubscripted operators $\delta$ and $\Delta$ denote the implicit and explicit temporal difference operators, respectively. Thus $\delta u_{i}^{n+1}$ is the temporal solution change calculated implicitly and $\Delta u_{i}^{n}$ is the temporal solution change calculated explicitly at mesh point $i$. The subscripted operators $\Delta_{+}$and $\Delta_{-}$denote spatial difference operators. Similarly, for $c \geq 0 \mathrm{Eq}$. (2) may be approximated by

$$
\left(1+\frac{\lambda \Delta t}{\Delta x}\right) \delta u_{i}^{n+1}=-\frac{c \Delta t}{\Delta x} \Delta_{-} u_{i}^{n}+\frac{\lambda \Delta t}{\Delta x} \delta u_{i-1}^{n+1}
$$

where $\delta u_{i}^{n+l}$ and $\lambda$ are shown above, and

$$
\Delta_{-} u_{i}^{n}=u_{i}^{n}-u_{i-1}^{n}
$$

A second-order-accurate two-step procedure similar to that of Eqs. (3) for $c<0$ or $c \geq 0$ is

$$
p:\left\{\begin{array}{l}
\Delta u_{i}^{n} \text { is determined as in Eq. (3) with } \nu=0 \\
\left(1+\frac{\lambda \Delta t}{\Delta x}\right) \delta u_{i}^{n+1}=\Delta u_{i}^{n}+\frac{\lambda \Delta t}{\Delta x} \delta u_{i+1}^{n+1} \\
u_{i}^{n+1}=u_{i}^{n}+\delta u_{i}^{n+1}
\end{array}\right.
$$

$$
c:\left\{\begin{array}{l}
\Delta u_{i}^{n+l} \text { is determined as in Eq. (3) with } v=0 \\
\left(1+\frac{\lambda \Delta l}{\Delta x}\right) \delta u_{i}^{n+1}=\Delta u_{i}^{n+1}+\frac{\lambda \Delta l}{\Delta x} \delta u_{i-1}^{n+1} \\
u_{i}^{n+1}=1 / 2\left(u_{i}^{n}+u_{i}^{n+1}+\delta u_{i}^{n+1}\right)
\end{array}\right.
$$

where $\lambda$ is chosen so that

$$
\lambda \geq \frac{I}{2}\left(|c|-\frac{\Delta x}{\Delta t}\right)
$$

Equations (4) are the implicit analogs of Eqs. (3) for $\nu=0$. The set of difference equations is unconditionally stable (in the sense that there is no restriction on the size of $\Delta l$ ). Each equation represents a bidiagonal scalar matrix equation and is solved by a single sweep through the mesh. For example, the solution of the predictor equation for $i=I, I-1, J-2, \ldots, 3,2,1$ is

$$
\delta u_{i}^{n+l}=\frac{\Delta u_{i}^{n}+(\lambda \Delta t / \Delta x) \delta u_{i+l}^{n+l}}{l+(\lambda \Delta l / \Delta x)}
$$

with boundary values specified for $u_{l+1}^{n}$ and $\delta u_{i+1}^{n+1}$.

We can unwrap the predictor step equation to gain some insight on its nature.

$$
\delta u_{i}^{n+1}=\frac{1}{I+\tau} \sum_{k=0}\left(\frac{\tau}{I+\tau}\right)^{k} \Delta u_{i+k}^{n}
$$

where

$$
\tau=\lambda \Delta t / \Delta x
$$

The change in value of the solution at mesh point $i$ as calculated by the predictor step of the explicit method of Eqs. 
(3) represents but the first term of this series with $\lambda=0$; the change in value calculated for the implicit method of Eq. (4) is determined by a positive weighted average of all the locally determined changes $\Delta u_{i+k}^{n}$, for $k=0,1,2, \ldots$ with the weights decaying with distance from mesh point $i$. Similarly, the change in value computed by the corrector step is determined by a positive weighted average of all the locally determined changes $\Delta u_{i-k}^{\overline{n+l} l}$ for $k=0,1,2 \ldots$.

The difference method represented by Eqs. (4) is secondorder accurate because the explicit method represented by Eqs. (3) is second-order accurate, and the net result of the terms added to Eqs. (3) to obtain Eqs. (4) are of third order; that is,

$$
\begin{aligned}
\delta u_{i}^{n+1} & =\Delta u_{i}^{n}+\tau\left(\delta u_{i+1}^{n+1}-\delta u_{i}^{n+l}\right) \\
& =\Delta u_{i}^{n}+\tau \Delta x \Delta t \frac{\partial(\partial u / \partial t)}{\partial x}+\mathcal{O}\left(\Delta t^{3}\right)
\end{aligned}
$$

and similarly

$$
\delta u_{i}^{n+1}=\Delta u_{i}^{n+1}-\tau \Delta x \Delta t \frac{\partial(\partial u / \partial t)}{\partial x}+\mathcal{O}\left(\Delta t^{3}\right)
$$

Thus

$$
\begin{aligned}
u_{i}^{n+1} & =1 / 2\left[u_{i}^{n}+\left(u_{i}^{n}+\delta u_{i}^{n+1}\right)+\delta u_{i}^{n+1}\right] \\
& =1 / 2\left[u_{i}+\left(u_{i}^{n}+\Delta u_{i}^{n}\right)+\Delta u_{i}^{n+1}\right]+\mathcal{O}\left(\Delta t^{3}\right) \\
& =1 / 2\left(u_{i}^{n}+u_{i}^{n+1}+\Delta u_{i}^{n+1}\right)+\mathcal{O}\left(\Delta t^{3}\right)
\end{aligned}
$$

Implicit Method, $c=0$

The Saul'ev method, ${ }^{2}$ modified somewhat, may be used to approximate $\mathrm{Eq}$. (2) with $\mathrm{c}=0$ :

$$
\begin{aligned}
& u_{i}^{n+1}=u_{i}^{n}+\frac{\nu \Delta t}{\Delta x^{2}}\left(u_{i+1}^{n}-2 u_{i}^{n}+u_{i-1}^{n}\right) \\
& +\frac{\lambda \Delta t}{\Delta x}\left[\left(u_{i+1}^{n+1}-u_{i+1}^{n}\right)-\left(u_{i}^{n+1}-u_{i}^{n}\right)\right]
\end{aligned}
$$

or

$$
\left(1+\frac{\lambda \Delta t}{\Delta x}\right) \delta u_{i}^{n+1}=\Delta u_{i}^{n}+\frac{\lambda \Delta t}{\Delta x} \delta u_{i+1}^{n+1}
$$

where

$$
\begin{gathered}
\delta u_{i}^{n+1}=u_{i}^{n+1}-u_{i}^{n} \\
\Delta u_{i}^{n}=\frac{\nu \Delta t}{\Delta x^{2}}\left(u_{i+1}^{n}-2 u_{i}^{n}+u_{i-1}^{n}\right)
\end{gathered}
$$

and for stability

$$
\lambda \geq \frac{1}{2}\left(\frac{2 v}{\Delta x}-\frac{\Delta x}{\Delta t}\right)
$$

Similarly, Eq. (2) may also be approximated by

$$
\left(1+\frac{\lambda \Delta t}{\Delta x}\right) \delta u_{i}^{n+1}=\Delta u_{i}^{n}+\frac{\lambda \Delta t}{\Delta x} \delta u_{i-1}^{n+l}
$$

where $\delta u_{i}^{n+1}, \Delta u_{i}^{n}$, and $\lambda$ are as before. Each approximation is unconditionally stable (unbounded $\Delta t$ ), first-order accurate in time, and second-order accurate in space under the constraint that $\nu \Delta t / \Delta x^{2}$ remains bounded as $\Delta t$ and $\Delta x$ approach zero. Each equation is also either upper or lower bidiagonal and can be solved by a single sweep through the mesh.
Implicit Method Applied to Eq. (2)

We may now present the implicit analog of the 1969 explicit method as follows:

$$
\begin{aligned}
& p:\left\{\begin{array}{l}
\Delta u_{i}^{n} \text { is determined as in Eq. (3) } \\
\left(1+\frac{\lambda \Delta t}{\Delta x}\right) \delta u_{i}^{n+1}=\Delta u_{i}^{n}+\frac{\lambda \Delta t}{\Delta x} \delta u_{i+1}^{n+1} \\
u_{i}^{n+j}=u_{i}^{n}+\delta u_{i}^{n+1}
\end{array}\right. \\
& c:\left\{\begin{array}{l}
\Delta u_{i}^{n+1} \text { is determined as in Eq. (3) } \\
\left(1+\frac{\lambda \Delta t}{\Delta x}\right) \delta u_{i}^{n+1}=\Delta u_{i}^{n+1}+\frac{\lambda \Delta t}{\Delta x} \delta u_{i-1}^{n+1} \\
u_{i}^{n+1}=1 / 2\left(u_{i}^{n}+u_{i}^{n+1}+\delta u_{i}^{n+1}\right)
\end{array}\right.
\end{aligned}
$$

where $\lambda$ is chosen so that

$$
\lambda \geq \max \frac{l}{2}\left\{|c|+\frac{2 v}{\Delta x}-\frac{\Delta x}{\Delta t}, \quad 0.0\right\}
$$

The method is unconditionally stable (unbounded $\Delta t$ ), requires solution of bidiagonal equations only, and is secondorder accurate under the constraint that $\nu \Delta t / \Delta x^{2}$ is bounded as $\Delta t$ and $\Delta x$ approach zero.

In Eqs. (5), either the predictor or the corrector steps are considered to consist of two stages. The first stage determines the local solution changes $\Delta u_{i}^{n}$ or $\Delta u_{i}^{n+1}$ by explicitly approximating the governing equations. The second stage uses the local solution changes in an implicit procedure, either upper or lower bidiagonal, to determine the actual solution changes $\delta u_{i}^{n+1}$ or $\delta u_{i}^{n+1}$ that are to be used to update the solution. Note that in the above inequality for choosing $\lambda$ we have avoided the selection of negative $\lambda$. There is no practical advantage for such a choice. On the other hand, if $\Delta t$ already satisfies the stability condition of the explicit method, the right side of the above inequality vanishes and $\lambda$ can be chosen to be zero. In this case, Eqs. (5) reduce to Eqs. (3). The implicit procedures of the second stage are not required for stability; thus the simpler explicit procedure suffices.

\section{Solution of the Navier-Stokes Equations} is

A desirable form for a numerical method for solving Eq. (1)

$$
\left.\begin{array}{rl}
\text { [numerics] } \delta U_{i, j}^{n+1} & =\text { [physics] } \\
U_{i, j}^{n+1} & =U_{i, j}^{n}+\delta U_{i, j}^{n+1}
\end{array}\right\}
$$

On the right-hand side, accurate local approximations are made to the governing physical equations. Thus, at each mesh point, values from near neighbors are used to obtain

$$
\{\text { physics }\}=\Delta U_{i, j}^{n}=-\Delta t\left(\frac{\Delta F}{\Delta x}+\frac{\Delta G}{\Delta y}\right)_{i, j}^{n}
$$

The responsibility of the left-hand side is to convey the locally determined solution changes globally in a stable manner without violating the laws of physics. In addition, for numerical efficiency the left-hand side should be as simple and straightforward as possible. To gain insight on how the solution changes are propagated, we differentiate Eq. (1) with respect to $t$ :

$$
\frac{\partial(\partial U / \partial t)}{\partial t}=-\frac{\partial A(\partial U / \partial t)}{\partial x}-\frac{\partial B(\partial U / \partial t)}{\partial y}
$$


where $A=\partial F / \partial U$ and $B=\partial G / \partial U$ are the Jacobians of $F$ and $G$. Thus the changes $\Delta t(\partial U / \partial t)$ should convect and diffuse throughout the flowfield according to Eq. (7), a closely related equation to Eq. (1). Implicitly approximating Eq. (7) in time, we have

$$
\left(I+\Delta t \frac{\partial A \cdot}{\partial x}+\Delta t \frac{\partial B \cdot}{\partial y}\right) \frac{\partial U^{n+1}}{\partial t}=\frac{\partial U^{n}}{\partial t}
$$

The dots in the equation indicate that the derivatives operate also on all the factors to the right. Defining

$$
\Delta U^{n}=\Delta T \frac{\partial U^{n}}{\partial t} \text { and } \delta U^{n+1}=\Delta t \frac{\partial U^{n+1}}{\partial t}
$$

we obtain

$$
\left(I+\Delta t \frac{\partial A \cdot}{\partial x}+\Delta t \frac{\partial B \cdot}{\partial y}\right) \delta U_{i, j}^{n+l}=\Delta U_{i, j}^{n}
$$

Therefore, the "numerics" should approximate the role of the factor in parentheses above with the use of numerically efficient procedures.

From the above considerations and from the basic numerical procedures of the last section, we can now present a numerical method for solving the equations of compressible viscous flow. Equation (1) may be numerically integrated in time by the following implicit predictor-corrector set of finitedifference equations:

$$
\begin{aligned}
& p:\left\{\begin{array}{l}
\Delta U_{i, j}^{n}=-\Delta t\left(\frac{\Delta_{+} F_{i, j}^{n}}{\Delta x}+\frac{\Delta_{+} G_{i, j}^{n}}{\Delta y}\right) \\
\left(I-\Delta t \frac{\Delta_{+}|A| \cdot}{\Delta x}\right)\left(I-\Delta t \frac{\Delta_{+}|B| \cdot}{\Delta y}\right) \delta U_{i, j}^{n+1}=\Delta U_{i, j}^{n} \\
U_{i, j}^{n+1}=U_{i, j}^{n}+\delta U_{i, j}^{n+1} \\
c:\left\{\begin{array}{l}
\Delta U_{i, j}^{n+1}=-\Delta t\left(\frac{\Delta_{-} F_{i, j}^{n+1}}{\Delta x}+\frac{\Delta_{-} G_{i, j}^{n+1}}{\Delta y}\right) \\
\left(I+\Delta t \frac{\Delta-|A| \cdot}{\Delta x}\right)\left(I+\Delta t \frac{\Delta_{-}|E| \cdot}{\Delta y}\right) \delta U_{i, j}^{n+1}=\Delta U_{i, j}^{n+1}
\end{array}\right. \\
U_{i, j}^{n+1}=1 / 2\left(U_{i, j}^{n}+U_{i, j}^{n+1}+\delta U_{i, j}^{n+1}\right)
\end{array}\right.
\end{aligned}
$$

where $\Delta_{+} / \Delta x, \Delta_{-} / \Delta x, \Delta_{+} / \Delta y$, and $\Delta_{-} / \Delta y$ are difference operators defined by

$$
\begin{array}{ll}
\frac{\Delta_{+} Z_{i, j}}{\Delta x}=\frac{Z_{i+1, j}-Z_{i, j}}{\Delta x} & \frac{\Delta_{-} Z_{i, j}}{\Delta x}=\frac{Z_{i, j}-Z_{i-1, j}}{\Delta x} \\
\frac{\Delta_{+} Z_{i, j}}{\Delta y}=\frac{Z_{i, j+1}-Z_{i, j}}{\Delta y} & \frac{\Delta_{-} Z_{i, j}}{\Delta y}=\frac{Z_{i, j}-Z_{i, j-1}}{\Delta y}
\end{array}
$$

As for the model equation, the first derivative terms are onesided differenced as shown above and the second derivative terms are centrally differenced. The matrices $|A|$ and $|B|$ have positive eigenvalues and are related to the Jacobians $A$ and $B$. Let $S_{x}, S_{y}$, and their inverses denote the matrices that diagonalize $A$ and $B$ with $\mu=\lambda=k=0$ (viscous terms neglected). If the gas equation of state is perfect, $p=(\gamma-$ 1) $\rho \epsilon, A=S_{x}^{-1} \Lambda_{A} S_{x}$, and $B=S_{y}^{-1} \Lambda_{B} S_{y}$, where

$S_{x}=\left[\begin{array}{rrrc}1 & 0 & 0 & -1 / c^{2} \\ 0 & \rho c & 0 & 1 \\ 0 & 0 & 1 & 0 \\ 0 & -\rho c & 0 & 1\end{array}\right]\left[\begin{array}{rrrr}1 & 0 & 0 & 0 \\ -u / \rho & 1 / \rho & 0 & 0 \\ -v / \rho & 0 & 1 / \rho & 0 \\ \alpha \beta & -u \beta & -v \beta & \beta\end{array}\right)$
$S_{y}=\left[\begin{array}{rrrr}1 & 0 & 0 & -1 / c^{2} \\ 0 & 1 & 0 & 0 \\ 0 & 0 & \rho c & 1 \\ 0 & 0 & -\rho c & 1\end{array}\right]\left[\begin{array}{rrrr}1 & 0 & 0 & 0 \\ -u / \rho & 1 / \rho & 0 & 0 \\ -v / \rho & 0 & 1 / \rho & 0 \\ \alpha \beta & -u \beta & -v \beta & \beta\end{array}\right)$

$\Lambda_{A}=\left(\begin{array}{cccc}u & 0 & 0 & 0 \\ 0 & u+c & 0 & 0 \\ 0 & 0 & u & 0 \\ 0 & 0 & 0 & u-c\end{array}\right), \Lambda_{B}=\left(\begin{array}{cccc}v & 0 & 0 & 0 \\ 0 & v & 0 & 0 \\ 0 & 0 & v+c & 0 \\ 0 & 0 & 0 & v-c\end{array}\right)$

and where $c=\sqrt{\gamma p / \rho}$ is the speed of sound, $\alpha=1 / 2\left(u^{2}+v^{2}\right)$ and $\beta=\gamma-1$.

The matrices $S_{x}$ and $S_{y}$ are each given above as the product of two matrices. For each, the right matrix represents a transformation from conservative to nonconservative variables, for example, from $(\delta \rho, \delta \rho u, \delta \rho v, \delta e)$ to $(\delta \rho, \delta u, \delta v$, $\delta p$ ). The left matrix transforms from nonconservative to characteristic form $\left(\delta \rho-\delta p / c^{2}, \rho c \delta u+\delta p, \delta v,-\rho c \delta u+\delta p\right)$ and $\left(\delta \rho-\delta p / c^{2}, \delta u, \rho c \delta v+\delta p,-\rho c \delta v+\delta p\right)$ for the $S_{x}$ and $S_{y}$ matrices, respectively. The inverses $S_{x}^{-1}$ and $S_{y}^{-1}$ are simple to derive. The matrices $|A|$ and $|B|$ are defined by

$$
|A|=S_{x}^{-1} D_{A} S_{x} \text { and }|B|=S_{y}^{-1} D_{B} S_{y}
$$

where

$$
\begin{aligned}
& D_{A}=\left(\begin{array}{cccc}
\lambda_{A 1} & 0 & 0 & 0 \\
0 & \lambda_{A 2} & 0 & 0 \\
0 & 0 & \lambda_{A 3} & 0 \\
0 & 0 & 0 & \lambda_{A d}
\end{array}\right), D_{B}=\left(\begin{array}{cccc}
\lambda_{B 1} & 0 & 0 & 0 \\
0 & \lambda_{B 2} & 0 & 0 \\
0 & 0 & \lambda_{B 3} & 0 \\
0 & 0 & 0 & \lambda_{B A}
\end{array}\right) \\
& \lambda_{A I}=\max \left\{|u|+\frac{2 \nu}{\rho \Delta x}-\frac{1}{2} \frac{\Delta x}{\Delta t}, \quad 0.0\right\} \\
& \lambda_{A 2}=\max \left\{|u+c|+\frac{2 \nu}{\rho \Delta x}-\frac{1}{2} \frac{\Delta x}{\Delta t}, \quad 0.0\right\} \\
& \lambda_{A 3}=\max \left\{|u|+\frac{2 v}{\rho \Delta x}-\frac{1}{2} \frac{\Delta x}{\Delta t}, \quad 0.0\right\} \\
& \lambda_{A 4}=\max \left\{|u-c|+\frac{2 \nu}{\rho \Delta x}-\frac{1}{2} \frac{\Delta x}{\Delta t}, \quad 0.0\right\} \\
& \lambda_{B I}=\max \left\{|v|+\frac{2 \nu}{\rho \Delta y}-\frac{1}{2} \frac{\Delta y}{\Delta t}, \quad 0.0\right\} \\
& \lambda_{B 2}=\max \left\{|v|+\frac{2 \nu}{\rho \Delta y}-\frac{1}{2} \frac{\Delta y}{\Delta t}, \quad 0.0\right\} \\
& \lambda_{B 3}=\max \left\{|v+c|+\frac{2 \nu}{\rho \Delta y}-\frac{l}{2} \frac{\Delta y}{\Delta t}, \quad 0.0\right\} \\
& \lambda_{B d}=\max \left\{|v-c|+\frac{2 \nu}{\rho \Delta y}-\frac{I}{2} \frac{\Delta y}{\Delta t}, \quad 0.0\right\} \\
& \nu=\max \left\{\mu, \quad \lambda+2 \mu, \quad \frac{\gamma \mu}{\text { Prandtl number }}\right\}
\end{aligned}
$$


Viscous effects are included through the use of the viscous coefficient $\nu$. For some test problems, this coefficient had to be increased during the initial part of the calculation when large transients in the solution occurred. This will be discussed in the next section.

For regions of the flow in which $\Delta t$ satisfies the following explicit stability conditions:

$$
\Delta l \leq \frac{1}{2} \frac{1}{(|u|+c) / \Delta x+\left(2 \nu / \rho \Delta x^{2}\right)}
$$

and

$$
\Delta t \leq \frac{l}{2} \frac{1}{(|v|+c) / \Delta y+\left(2 v / \rho \Delta y^{2}\right)}
$$

all $\lambda_{A}$ and all $\lambda_{B}$ vanish and the set of difference Eqs. (8) reduces to the 1969 explicit equations with simple solution. For other regions in which neither relation is satisfied, the resulting difference equations are either upper or lower block bidiagonal equations with fairly straightforward solutions. For example, let us solve the predictor step of Eqs. (8) assuming $\Delta t$ satisfies neither of Eqs. (9). If $\delta U_{i, j}^{*}$ denotes

$$
\left(I-\Delta t \frac{\Delta_{+}|B|}{\Delta y}\right) \delta U_{i, j}^{n+1}
$$

the predictor step becomes

$$
\left(I+\left.\frac{\Delta t}{\Delta x}|A|\right|_{i, j} ^{n}\right) \delta U_{i, j}^{*}=\Delta U_{i, j}^{n}+\frac{\Delta t}{\Delta x}|A|_{i+1, j}^{n} \delta U_{i+1, j}^{*}
$$

and upper bidiagonal equation. The solution can be obtained for each $j$ by sweeping in the decreasing $i$ direction. After obtaining $\delta U_{i, j}^{*}$ for all $i, j$, then

$$
\left(I+\frac{\Delta t}{\Delta y}|B|_{i, j}^{n}\right) \delta U_{i, j}^{n+\prime}=\delta U_{i, j}^{*}+\frac{\Delta t}{\Delta y}|B|_{i, j+1}^{n} \delta U_{i, j+1}^{n+1}
$$

is solved. This equation is also upper bidiagonal and is solved for each $i$ by sweeping in the decreasing $j$ direction, thus obtaining $\delta U_{i, j}^{n+l}$ for all $i, j$. Then $U_{i, j}^{n+1}=U_{i, j}^{n}+\delta U_{i, j}^{n+1}$, etc. Some discussion on inverting the matrices $(I+(\Delta t / \Delta y)$ $|A|\}$ and $\{I+(\Delta t / \Delta y)|B|\}$ and on boundary conditions for $\delta U$ will be given in the next section.

The above method is stable for unbounded $\Delta t$, is written in conservation-law form, and is second-order accurate under the condition that $\nu \Delta t / \rho \Delta x^{2}$ and $\nu \Delta t / \rho \Delta y^{2}$ remain bounded as $\Delta t, \Delta x$, and $\Delta y$ approach zero. This method is more efficient than existing methods for solving the equations of compressible viscous flow because 1) for regions of the flow satisfying explicit stability criteria, the method requires no more computer time than an explicit method, and 2) for the other regions, straightforward block bidiagonal equations need only be solved rather than the more costly block tridiagonal equations of competing methods. Also, the method propagates and diffuses change determined locally using propagation speeds and kinematic viscosities near in magnitude to those of the governing equations themselves. No additional parameters not physically relevant are used. In this sense the method approximates the desired form of Eq. (6).

\section{Program Notes}

To provide a better understanding of the method, let us examine the procedure for solving the block bidiagonal equation for the $y$-coordinate direction,

$$
\left[I+\frac{\Delta t}{\Delta y}|B|_{i, j}^{n}\right] \delta U_{i, j}^{n+1}=\delta U_{i, j}^{*}+\frac{\Delta t}{\Delta y}|B|_{i, j+1}^{n} \delta U_{i, j+1}^{n+1}
$$

The procedure begins with the vectors $U_{i, j}^{n}$, given for all $i=1,2, \ldots, I$ and $j=1,2, \ldots, J ; \delta U_{i, j}^{*}$ given for all $i=2,3, \ldots, I-1$ and $j=2,3, \ldots, J-1$; and $|B|{ }_{i, j}^{n} \delta U_{i, j}^{n+l}$ given for all $i=$ $2,3, \ldots, I-1$. The quantity $|B| \eta, \delta U_{i, j^{\prime}}$ represents the flux of change that crosses the top mesh boundary. If this boundary is located in the far flowfield that remains unchanged during the calculation, as in the case of the test problems to be discussed later, this flux is set equal to zero. Otherwise it must be specified by suitable boundary conditions.

Using the defined quantities of the last section, the procedure is as follows. For each $i$ and for $j=J-1$, $J-2, \ldots, 3,2$,

$$
\begin{aligned}
& \text { 1) } W=\delta U_{i, j}^{*}+\frac{\Delta t}{\Delta y}|B|_{i, j+1}^{n} \delta U_{i, j+1}^{n+1} \\
& \text { 2) } X=S_{y} W \\
& \text { 3) } D_{B} \text { is calculated } \\
& \text { 4) } Y=\left(I+\frac{\Delta t}{\Delta y} D_{B}\right)^{-1} X \\
& \text { 5) } \delta U_{i, j}^{n+1}=S_{y}^{-1} Y \\
& \text { 6) } Z=D_{B} Y \\
& \text { 7) }|B| \delta U_{i, j}^{n+1}=S_{y}^{-1} Z
\end{aligned}
$$

Each of the above seven steps requires approximately four Fortran statements. The matrix inversion of step 4 is trivial because the matrix is diagonal. The solution at grid point $i, j$ is obtained at step 5, and the flux to be used in the calculation at grid point $i, j-1$ is obtained at step 7 . If the lower boundary is a wall or a plane of symmetry, as in the case of the test problems to be discussed, the computed end flux terms, $|\boldsymbol{B}| \delta U_{i, 2}^{n+1}$, are saved to be used as a boundary condition for the corrector step that sweeps away from this boundary in the increasing $j$ direction. According to the usual rules of reflection, the starting flux of the corrector step is given by

$$
|B| \delta U_{i, i}^{n+1}=E|B| \delta U_{i, 2}^{n+1}
$$

where

$$
E=\left(\begin{array}{rrrr}
1 & 0 & 0 & 0 \\
0 & 1 & 0 & 0 \\
0 & 0 & -1 & 0 \\
0 & 0 & 0 & 1
\end{array}\right)
$$

This condition assures that the net mass, tangential momentum, and energy fluxes transmitted across this boundary vanish and that the net transverse momentum at the boundary remains zero.

The elements of $S_{y}^{-1}, D_{B}$, and $S_{y}$ are evaluated at each grid point using local values. If during the initial part of the calculation the variables change rapidly in time, the evaluation of the elements $\lambda_{B}$ as given in the last section may not be adequate to maintain stability. In particular, the viscous coefficient term $2 \nu / \rho \Delta y$ may be underestimated. Thus, for some of the higher Reynolds number test case problems, in addition to this term, the following term was used:

$$
T=\frac{\left|\left(\delta p / c^{2}\right)-\delta \rho\right|}{(\Delta t / \Delta y)[(\gamma-I) / \gamma] \rho}
$$

This term represents a measure of the change in entropy caused primarily by viscosity. It is evaluated during step 3 


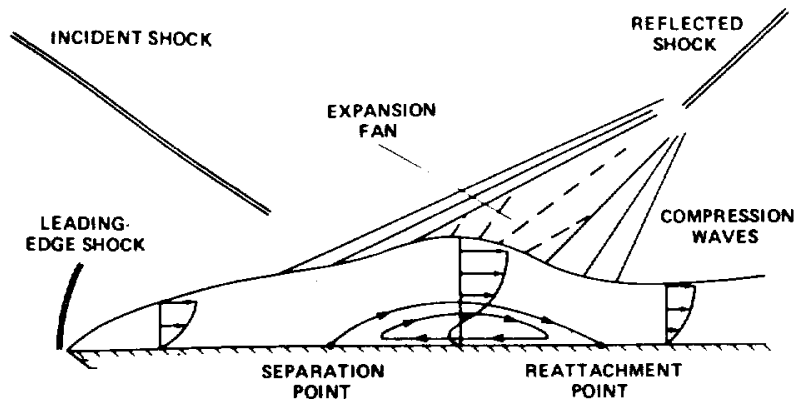

Fig. 1 Skelch of shock/boundary-layer interaction.

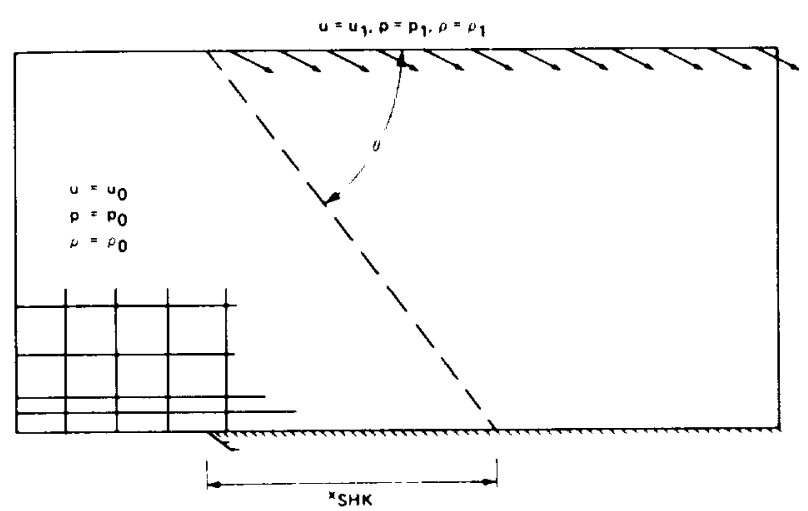

Fig. 2 Initial flowfield for shock/boundary-layer interaction.

using the first element of the vector $X$,

$$
T=\frac{\left|x_{1}\right|}{(\Delta t / \Delta y)[(\gamma-I) / \gamma] \rho}
$$

As steady state is approached, $\left|x_{1}\right|$ approaches zero and the added term vanishes.

The number of Fortran statements for the above seven steps is approximately 30 . Approximately 50 are required for a complete subroutine that includes index control so that sweeps can be made in either direction using the same statements, calculation of additional useful intermediate variables, setting of boundary conditions, and a test to see if the seven steps should be done at all, at each grid point, by checking to see if the time step size is larger than that allowed by the explicit stability criteria of the last section.

The implicit procedure outlined above for the $y$-coordinate direction sweeps first toward the lower boundary in the predictor, then sweeps away from this boundary in the corrector using reflected boundary conditions. The implicit procedure for the $x$-coordinate direction is similar except that the sweep directions for the predictor and corrector steps should be switched at each new time step. For the test problems of the next section, the flux of change at the start of each sweep in the $i$ direction was set to zero because either the flow at the boundary was steady or the mesh spacing was large enough so that the elements of $D_{A}$ and hence $|A|$ vanished.

\section{Numerical Results}

To test the numerical method just presented, a series of shock-wave/boundary-layer interaction problems were calculated. The flowfield representing this interaction is sketched in Fig. 1. The sketch shows an externally generated shock wave incident upon a boundary layer on a flat plate. If the shock wave is strong enough, the boundary layer will, as shown in the sketch, separate from the surface of the plate and reattach downstream. Between the separation and reattachment points there is a region of rotating fluid that causes the boundary layer to thicken and generate a series of compression and expansion waves that eventually form the reflected shock wave. The separation region is fairly sensitive to calculate and therefore serves as a good test for a numerical method.

The initial flowfield and boundary conditions are shown schematically in Fig. 2. The initial condition for the interior of the flowfield is uniform flow. The flow variables at the top mesh boundary were set to either freestream values or values for a given shock strength so that the shock wave would impinge on the plate surface at the lower boundary at a given point. The lower surface was either a plane of symmetry or a wall surface, and reflective boundary conditions were used $\left(u_{i, 1}=u_{i, 2}\right.$ ahead of the leading edge of the plate and $-u_{i, 2}$ along the plate surface; $v_{i, 1}=-v_{i, 2}, p_{i, 1}=p_{i, 2}$, and $\epsilon_{i, 1}=\epsilon_{i, 2}$ ahead of the leading edge and along the plate for an adiabatic wall; or $2 \epsilon_{\text {wall }}-\epsilon_{i, 2}$ for an isothermal wall where $\epsilon_{\text {wall }}$ is the internal energy of the gas at the wall). For the selected test case problems, the freestream was supersonic and therefore the values at the upstream boundary were held fixed. At the downstream or exit boundary, zero-order extrapolation $\left(U_{t, j}=U_{t-1, j}\right)$ was used. The flow leaving is either parabolic (near the plate) or hyperbolic (away from the plate) in the streamwise direction so that errors made here should not propagate upstream. Tests using more accurate boundary treatment have verified that this is true.

The mesh contained $32 \times 32$ points and could be stretched in both the $x$ and $y$ directions. The program has the capability to calculate for a given number of time steps and then redistribute the mesh points in a smaller "focused" region and continue running. Approximately 16 mesh points spanned the boundary layer. The time step was chosen initially so that the freestream moved a given percentage (approximately $1 \%$ ) of the plate length during each time step. For high Reynolds number calculations in which $\nu \Delta l / \rho \Delta y^{2}>1 / 2$, the time step was successively reduced near the end of the calculation to avoid any possible steady-state solution dependence on $\Delta t$.

The calculated results for laminar Mach 2 flow at a Reynolds number of $2.96 \times 10^{5}$ are shown in Figs. 3a-c. A shock wave incident upon the plate increased surface pressure by a factor of 1.4 and was sufficient to cause flow separation. Molecular viscosity was calculated using Sutherland's formula. The calculation ran for 256 time steps, at which time the flow was near steady state, and then the mesh was rezoned to cover just the interaction region and run an additional 256 steps. The calculation required about $1.5 \mathrm{~min}$ of computer time on a CDC 7600 computer. The calculated surface pressure is compared with the experimental measurements of Hakkinen ${ }^{3}$ in Fig. 3a and with boundary-layer theory ${ }^{4}$ (in the absence of a shock) for skin friction in Fig. 3b. The experimental probes in the separated region were unable to measure skin friction other than to show that it was zero or negative. The calculated velocity profiles ahead, aft, and in the separation region are compared with the calculated results of the 1969 explicit method in Fig. 3c. The calculated results of the new method are consistent with experiment, theory, and other numerical results.

Table 1 compares the timings of the new method with those of the 1969 method for a series of shock interaction problems. Each problem was calculated to the same physical time, which for the new method required 256 time steps. A simple algebraic eddy viscosity model was used in the turbulent flow calculations. For each case the table shows the Reynolds number, the ratio of the time step used to the maximum allowed by explicit stability conditions (CFL), the computer time required per step per grid point on a CDC 7600 computer, and the total computer time required. As the table shows, the new method is very efficient. It is, however, only slightly more efficient than the hybrid explicit-implicit- 

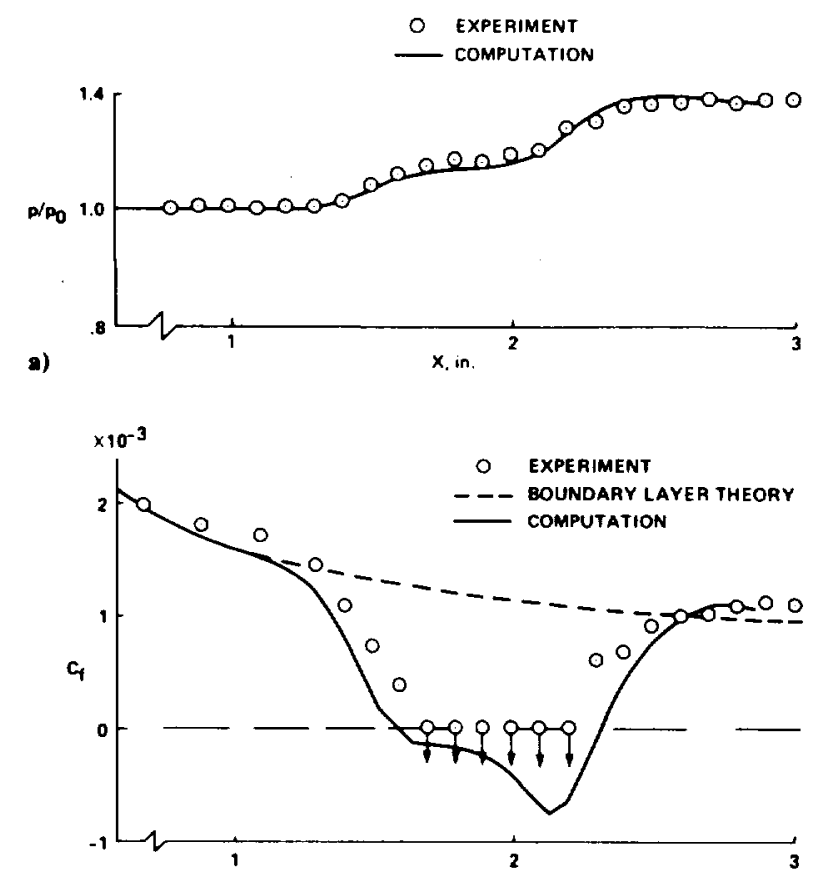

b)

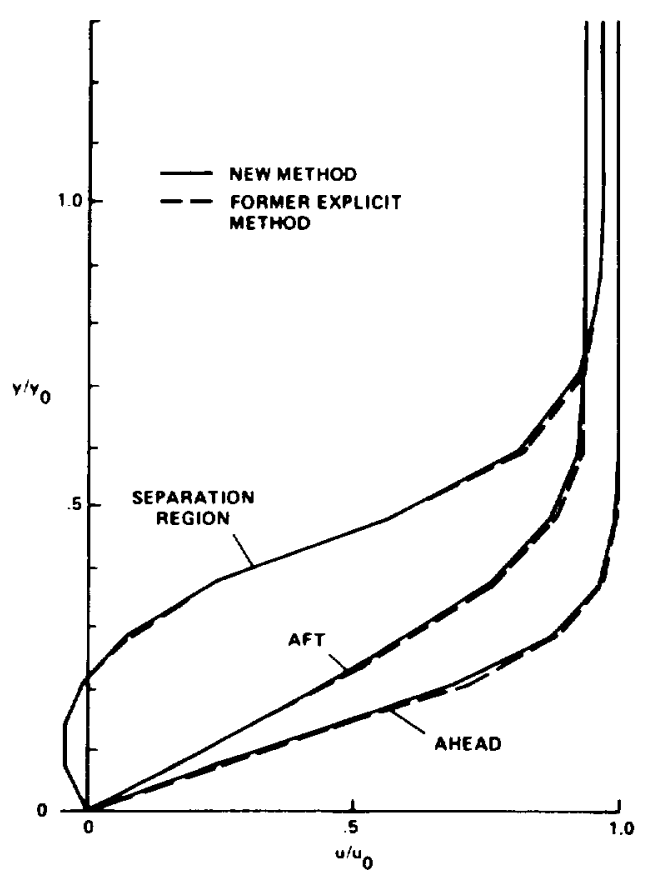

c)

Fig. 3 Comparison of resul(s: a) surface pressure, b) skin friction, c) velocity profiles.
Table 1 Computation time

\begin{tabular}{lcccc}
\hline \hline Case & Method & CFL & $\begin{array}{c}\text { CDC 7600 time, } \\
\text { step grid pt } \\
\times 10^{-4} \mathrm{~s}\end{array}$ & $\begin{array}{c}\text { Total } \\
\text { time }\end{array}$ \\
\hline Laminar & 1969 & 0.9 & 1.25 & $12 \mathrm{~min}$ \\
$R=3 \times 10^{5}$ & New & 20 & 1.55 & $41 \mathrm{~s}$ \\
Turbulent & 1969 & 0.9 & 1.55 & $2 \mathrm{~h}$ \\
$R=3 \times 10^{6}$ & New & 160 & 1.85 & $48 \mathrm{~s}$ \\
Turbulent & 1969 & 0.9 & 1.55 & $15 \mathrm{~h}$ \\
$R=3 \times 10^{7}$ & New & 1200 & 1.85 & $48 \mathrm{~s}$ \\
\hline \hline
\end{tabular}

${ }^{\mathrm{a}}$ Estimated.

characteristic method' the author presented in 1976 for the lower Reynolds number calculations and, at most, twice as fast for the higher Reynolds number cases, although it is an order of magnitude easier to program. It is also, for the test cases considered, more than twice as fast per time step per grid point as the block tridiagonal implicit methods in use today. Part of the reason for this is that, because of the mesh point spacing, more than half the total number of mesh points required only use of the 1969 explicit method (the first stage of the method) of solution. At these points, the implicit procedures (the second stage of the method) were skipped. For other calculations requiring more or less use of the implicit procedures, the computer time per step per grid point would be more or less than the values given in the table. The estimated maximum for a two-dimensional calculation is $2.45 \times 10^{-4} \mathrm{~s}$ for laminar flow and $2.75 \times 10^{-4} \mathrm{~s}$ for turbulent flow using an algebraic eddy viscosity model.

\section{Conclusions}

A new method has been presented for solving the equations of compressible viscous flow. For many applications this method is more efficient and easier to program than other methods in use today.

The method is the implicit analog of the explicit method that the author presented in 1969. Because the new method uses the 1969 method as its first stage, many exisiting computer programs also using the 1969 method can be updated by adding the described implicit procedures that form the second stage.

\section{References}

'MacCormack, R. W., "The Effect of Viscosity in Hypervelocity Impact Cratering." AlAA Paper 69-354, 1969.

${ }^{2}$ Richtmyer, R. D. and Morton, K. W., Difference Methods for Initial Value Problems, 2nd ed., Wiley Interscience, New York, 1969.

${ }^{3}$ Hakkinen, R. J., Greber, I., Trilling, L., and Abarbanel, S. S., "The Interaction of an Oblique Shock Wave with a Laminar Boundary Layer," NASA Memo 2-18-59W, 1959.

"van Driest, E. R., "Investigation of Laminar Boundary Layers in Compressible Fluids Using the Crocco Method," NACA TN 2597. Jan. 1952.

"MacCormack, R. W.. "An Efficient Numerical Method for Solving the Time-Dependent Compressible Navier-Stokes Equations at High Reynolds Number," Proceedings of the ASME Winter Annual Meeting, ASME, New York, 1976, pp. 49-64; also NASA TMX-73129. 